\title{
A Single Multi-Task Deep Neural Network with Post-Processing for Object Detection with Reasoning and Robotic Grasp Detection
}

\author{
Dongwon Park ${ }^{1}$, Yonghyeok Seo ${ }^{1}$, Dongju Shin, Jaesik Choi and Se Young Chun*
}

\begin{abstract}
Recently, robotic grasp detection (GD) and object detection (OD) with reasoning have been investigated using deep neural networks (DNNs). There have been works to combine these multi-tasks using separate networks so that robots can deal with situations of grasping specific target objects in the cluttered, stacked, complex piles of novel objects from a single RGB-D camera. We propose a single multi-task DNN that yields the information on GD, OD and relationship reasoning among objects with a simple post-processing. Our proposed methods yielded state-of-the-art performance with the accuracy of $98.6 \%$ and $74.2 \%$ and the computation speed of 33 and 62 frame per second on VMRD and Cornell datasets, respectively. Our methods also yielded $95.3 \%$ grasp success rate for single novel object grasping with a 4 -axis robot arm and $86.7 \%$ grasp success rate in cluttered novel objects with a Baxter robot.
\end{abstract}

\section{INTRODUCTION}

Robot grasping of particular target objects in cluttered, stacked and complex piles of novel objects is a challenging open problem. Humans instantly identify / locate target objects and their nearby objects (object detection or OD), figure out location-wise relationship among objects (reasoning), and detect multiple grasps of the targets and their associated objects (grasp detection or GD). However, these tasks are still quite challenging for robots. Locating the targets and nearby objects in the piles of objects, reasoning their relationships and detecting multiple robotic grasps accurately and quickly are important multi-tasks for successful robotic grasping.

Deep learning based approaches have been actively investigated for robot grasp detection since the work of Lenz et al. [3], [4]. Thanks to the Cornell robotic grasp detection open database [3] and the advance of deep learning techniques, there have been many works proposed [5], [6], [7] and current state-of-the-art GD accuracy on the Cornell dataset is up to $97.7 \%$ [8]. The Cornell dataset contains images with a single object and multiple grasp labels. Deep neural networks (DNNs) trained with this data generally yielded multiple grasps for a single object or multiple objects that are separately placed. Due to the capacity of DNNs, they often yielded good GD results for novel objects. However, these DNNs with the Cornell dataset do not seem general enough for the task of robotic grasping in the cluttered piles of objects. Moreover, it is especially challenging which object the robot has to grasp first in order not to damage other objects for cluttered or stacked objects or in order to efficiently grasp specific target objects. In other words, a

Dongwon Park, Yonghyeok Seo, Dongju Shin and Se Young Chun are with School of Electrical and Computer Engineering, UNIST, Ulsan, Republic of Korea. Jaesik Choi is with Graduate School of Artificial Intelligence, KAIST, Daejeon, Republic of Korea.

${ }^{1}$ Equal contribution. *Corresponding author: sychun@unist.ac.kr robot must know if an object is on another object in the piles of objects for successful grasping.

Recently, Zhang et al. proposed multi-task convolution robotic grasping networks to address the problem of combining GD and OD with relationship reasoning in the piles of objects [1]. This method consists of several DNNs that are responsible for generating local feature maps, GD, OD and relationship reasoning separately. More specifically, features are extracted using ResNet-101 based region proposal network (RPN) and then are fed into three DNNs corresponding to three tasks: OD, GD and relationship prediction among objects to perform grasping considering relationships and orderings (reasoning). This approach allowed easy matching for reasoning and achieved high GD accuracy of $70.1 \%$ on the VMRD robot grasping dataset [9] with reasonable computation speed of 6.5 frame per second (FPS). However, this modular structure could be further optimized and improved for higher accuracy, faster computation speed and less DNNs for potentially reduced GPU memory usage.

In this paper, we propose a single multi-task DNN with a simple post-processing for OD with reasoning and GD

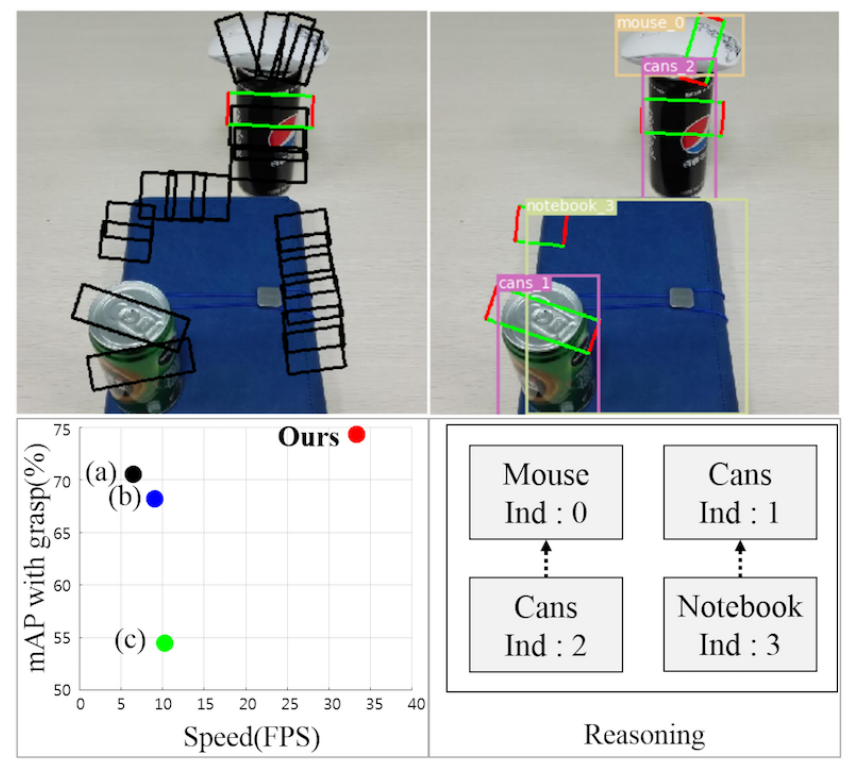

Fig. 1: (top left panel) GD with grasp candidates (black rectangles) and the best grasp (green and red rectangle) (right panels) multi-tasks of GD, OD and relationship reasoning. (bottom left panel) computation speed (FPS) vs. prediction accuracy (mAP) for multi-task grasping detection of our method achieving state-of-the-art performance in both accuracy and speed and other previous works of (a) [1], (b,c) [2]. 
on the piles of novel objects using the information from a single RGB-D camera. Our proposed methods extended YOLOv3 [10], a state-of-the-art OD method, to dealing with multi-tasks of OD, GD and relationship reasoning, but maintained its simple single network structure. Ablation studies were performed to further optimize different components of our multi-task networks. Our methods yielded state-of-the-art multi-task GD performance $(74.2 \%, 98.6 \%)$ on the VMRD and Cornell datasets, respectively, with realtime computation speed (30 and 62 FPS) for high-resolution images of $608 \times 608$ and $320 \times 320$ as illustrated in Fig. 1 . Our methods were also applied to real robotic grasping tasks with a 4 axis robot arm on single novel objects as well as a Baxter on multiple novel objects in various settings of piles (cluttered, stacking, invisible scenes) and yielded $95.3 \%$ grasp success rate for single novel object grasping and $86.7 \%$ grasp success rate in cluttered novel objects, respectively.

\section{RELATED WORK}

Pre-deep learning era. Data-driven GD for novel objects has been investigated extensively [11]. Saxena et al. proposed a machine learning (ML) based method to rank the best graspable location for all candidate image patches from different locations [12]. Jiang et al. proposed a 5D robotic grasp representation by using a ML method to rank the best graspable image patch whose representation includes orientation and gripper distance among all candidates [13]. Two-stage classification based approach. Lenz et al. proposed to use a sparse auto-encoder (SAE) to train the network to rank the best graspable candidate image patch from sliding window with RGB-D [3], [4]. Wang et al. proposed a realtime classification based GD method using a stacked SAE for classification with efficient grasp candidates generation [14]. Mahler et al. proposed Dex-Net 2.0 with GQ-CNN that estimates grasps for parallel grippers from a single depth image trained with synthetic point-cloud data [15].

Single-stage regression based approach. Redmon et al. proposed a deep learning regressor GD method based on the AlexNet [16] with fast computation time [5]. When performing robotic grasp regression and object classification together, image-wise prediction accuracy was able to be improved without increasing computation time. Kumra et al. also proposed a real-time regression based GD method using ResNet [17] especially for RGB-D and this work yielded improved performance with fast computation time.

Multibox based approach. Redmon et al. also proposed a multibox GD method (called MultiGrasp) by dividing the input image into $\mathrm{S} \times \mathrm{S}$ grid and applying regression based GD to each grid box [5]. This approach did not increase computation time but increased prediction accuracy for multiobject, multigrasp detection. Guo et al. proposed a hybrid multibox approach with visual and tactile information by classifying graspability, angles $(\theta)$, and by regressing locations and grasp width $(w)$, height $(h)$ [6]. Chu et al. proposed two-stage neural networks combining grasp region proposal network (RPN) and robotic GD network [7]. Zhou et al. proposed rotational anchor box and angle matching (angle classification + regression) to further improve prediction accuracy [8]. This method is currently state-of-the-art in GD on the Cornell dataset with computation time of $117 \mathrm{~ms}$ per image $(320 \times 320)$. Note that the networks of Guo [6], Chu [7] and Zhou [8] are based on faster-RCNN [18].

Hybrid approach. Asif et al. proposed GraspNet that predicts graspability and then estimates robotic grasp parameters based on high-resolution grasp probability map [19].

Depth vs color information. There are several works that use depth information only or color information only for GD. Johns et al. developed a method to estimate a grasp score (quality) from a single depth image [20]. Dex-Net 3.0 was proposed to estimate robotic grasps for suctions from a depth image (point cloud) trained with synthetic data [21]. There have been a couple of works to use depth images only for closed-loop grasping [22], [23]. Morrison et al. demonstrated that using fast, lightweight neural network was important for grasping dynamic objects [23]. There also have been some works using color images only for GD. Since depth image is often quite noisy [24], only RGB images have been used for learning 5D grasp representation from a color image [25] and for achieving almost state-of-the-art performance [8].

OD with GD. Zhang et al. proposed a VMRD grasping dataset with object detection and object relation and a Visual manipulation relationship network (VMRN) [9]. Based on SSD [26], an OD method, VMRN extracted features and then predicted relationship of objects. Zhang et al. further developed multi-task robotic grasp networks for OD, GD and reasoning with VMRN [2], [1] based on the GD work of Zhou [8] for grasping tasks in complex piles of objects.

\section{PROPOSED METHODS}

\section{A. Problem description}

Single object robot grasping A 5D robotic grasp representation is widely used for GD with a parallel gripper when a single 2D image (RGB or RGB-D) is used [13], [4]. This representation is a vector of $\left\{x_{g d}, y_{g d}, \theta_{g d}, w_{g d}, h_{g d}\right\}$ that consists of location $\left(x_{g d}, y_{g d}\right)$, orientation $\theta_{g d}$, gripper opening width $w_{g d}$ and parallel gripper plate size $h_{g d}$.

\section{Multi-task robot grasping}

Grasping a specific target object in cluttered and stacking objects requires more than single object grasping information and needs additional information such as object class and relationship reasoning (see Fig. 1) for sequential grasp planning. We extended the previous 5D robotic grasp representation to including object class and stacking order among objects as follows:

$$
\left\{x_{g d}, y_{g d}, \theta_{g d}, w_{g d}, h_{g d}, c l s_{g d}, \operatorname{ord}_{g d}\right\} .
$$

\section{B. Reparametrization of $15 D$ representation}

We propose a $15 \mathrm{D}$ representation for multi-task robot grasping problem to exploit a single multi-task DNN for $\mathrm{OD}, \mathrm{GD}$ and reasoning altogether. The parameters for OD are $\left\{x_{o d}, y_{o d}, w_{o d}, h_{o d}, c l s_{o d}, p r_{o d}\right\}$ and the parameters for GD are $\left\{x_{g d}, y_{g d}, w_{g d}, h_{g d}, c l s_{g d}, p r_{g d}, \theta_{g d}\right\}$. where $p r_{o d}$ is a probability of an object existing and $p r_{g d}$ is a graspable 
probability. The parameters of reasoning are $\left\{c l s_{f c}, c l s_{c c}\right\}$ for ordering objects $\left(o r d_{g d}\right)$. Father class (FC) and children class (CC) are labels under and over the predicted target object, respectively. FC and CC are predicted of each grid.

We propose the following reparametrization of OD and GD for robotic grasping in the piles of objects:

$$
\begin{array}{r}
O D=\left\{t_{o d}^{x}, t_{o d}^{y}, t_{o d}^{w}, t_{o d}^{h}, t_{o d}^{p r}, t_{o d}^{c l s}\right\}, R=\left\{t_{f a}^{c l s}, t_{c c}^{c l s}\right\} \\
G D=\left\{t_{g d}^{x}, t_{g d}^{y}, t_{g d}^{w}, t_{g d}^{h}, t_{g d}^{p r}, t_{g d}^{c l s}, t_{g d}^{\theta}\right\}
\end{array}
$$

where $x_{j}=\sigma\left(t_{j}^{x}\right)+c_{j}^{x}, y_{j}=\sigma\left(t_{j}^{y}\right)+c_{j}^{y}, \sigma(\cdot)$ is a sigmoid function, $w_{j}=p_{j}^{w} \exp \left(t_{j}^{w}\right), h_{j}=p_{j}^{h} \exp \left(t_{j}^{h}\right), \theta_{g d}=p_{g d}^{\theta}+$ $t_{g d}^{\theta}, c l s_{j}=\operatorname{softmax}\left(t_{j}^{c l s}\right), c l s_{r s}=\sigma\left(t_{r s}^{c l s}\right), p r_{j}=\sigma\left(t_{j}^{p r}\right)$, $j \in\{o d, g d\}$ and $r s \in\{f c, c c\}$. Note that $p_{j}^{h}, p_{j}^{w}$ and $p_{g d}^{\theta}$ are the pre-defined height, width, orientation of an anchor box, respectively, and $\left(c_{j}^{x}, c_{j}^{y}\right)$ are the location of the top left corner of each grid cell (known). Thus, DNN for GD of our proposed methods will estimate $\left\{t_{j}^{x}, t_{j}^{y}, t_{j}^{\theta}, t_{j}^{w}, t_{j}^{h}, t_{j}^{p r}\right\}$ instead of $\left\{x_{j}, y_{j}, \theta_{g d}, w_{j}, h_{j}, p r_{j}\right\} . x_{j}, y_{j}, w_{j}, h_{j}$ are properly normalized so that the size of each grid is $1 \times 1$. Lastly, the angle $\theta_{g d}$ will be modeled as a discrete and continuous value instead of a continuous value.

Anchor box: $\mathbf{w}, \mathbf{h}$ in each cell. Anchor box approach has been used for OD [27]. Due to re-parametrization with anchor box, estimating $w_{j}, h_{j}$ is converted into estimating $t_{j}^{w}, t_{j}^{h}$, which are related to the expected values of various sizes of $w_{j}, h_{j}$. Then, the best grasp representation among all anchor box candidates is selected for the final output. Thus, re-parametrization changes regression problem into regression + classification problem for $w_{j}, h_{j}$.

Anchor box: orientation in each cell. While MultiGrasp took regression approach for $\theta_{g d}$ [5], Guo et al. converted regression problem of estimating $\theta_{g d}$ into the classification for $\theta_{g d}$ among finite number of angle candidates in $\{0, \pi / 18, \ldots, 17 \pi / 18\}$ [6]. Zhang [28] proposed orientation anchor box so that the angle is determinded using classification as well as discrete anchor box rotations. Mean average precision (mAP) increased by $3 \%$ when using orientation anchor box (4 angles) over angle classification on the VMRD.

Object class: cls in each cell. When objects are stacked in a complex way, it becomes a difficult task to match OD result (detection bounding box) with GD result without additional information such as object classes. For this task, object class is predicted for each of grasp detection box result so that our proposed model can yield grasping detection boxes, their grasping points and corresponding object classes. A softmax was selected for class activation function through our selfevaluation ablation study that will be reported shortly.

FC and $\mathrm{CC}$ in each cell. For inter-object relationship, we propose to predict FC and CC along with other detection results. FC and $\mathrm{CC}$ are class labels under and over the target object, respectively. FC and $\mathrm{CC}$ consist of object class labels and no-class label. $\left(t_{f c}^{c l s}, t_{c c}^{c l s}\right) \in$ $\left\{\right.$ class $_{1}$, class $_{2}, \ldots$, class $\left._{\text {none }}\right\}$. In our experiment, we observed that $\mathrm{CC}$ is more accurately estimated than FC. Thus, we only used $\mathrm{CC}$ for reasoning for the best possible results.

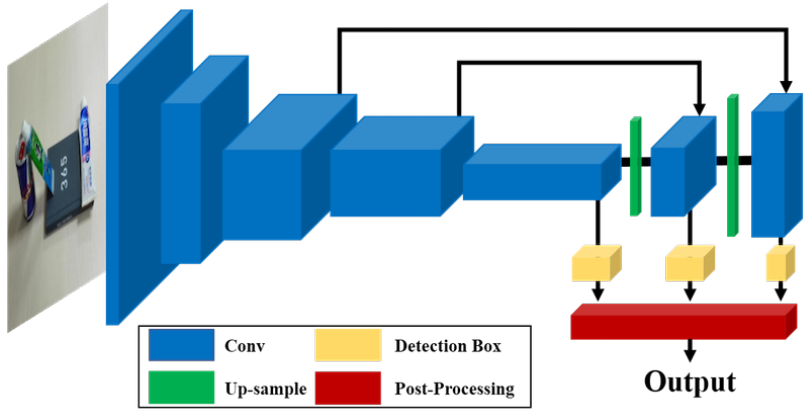

Fig. 2: Proposed FCNN architecture based on Darknet.

\section{Proposed FCNN with predictions across scales}

Our proposed FCNN inherited pre-trained Darknet-53 of YOLOv3 for OD [10] and extended it for multi-task OD with reasoning and GD as illustrated in Fig. 2. For our multitask predictions, we did not only adopted prediction across scales for OD using feature pyramid networks [29], but also extended it for reasoning and GD.

On the low-resolution scale, three anchor boxes $(w, h)$ for OD 1 anchor box for GD and 4 anchor boxes for grasping angles are predicted as

$$
\begin{aligned}
& \left(p_{o d}^{w}, p_{o d}^{h}\right) \in\{(540,540),(480,480),(420,420)\}, \\
& \left(p_{g d}^{w}, p_{g d}^{h}\right) \in\{(300,300)\}, p_{g d}^{\theta} \in\{0, \pi / 4,2 \pi / 4,3 \pi / 4\} .
\end{aligned}
$$

Then, on the mid-resolution scale after $\times 2$ bilinear upsampling, 3 anchor boxes for OD, 1 anchor box for GD and 4 anchor boxes for grasping angles are estimated as

$$
\begin{aligned}
& \left(p_{\text {od }}^{w}, p_{\text {od }}^{h}\right) \in\{(360,360),(300,300),(240,240)\}, \\
& \left(p_{g d}^{w}, p_{g d}^{h}\right) \in\{(100,100)\}, p_{g d}^{\theta} \in\{0, \pi / 4,2 \pi / 4,3 \pi / 4\} .
\end{aligned}
$$

On the high-resolution scale after $\times 4$ up-sampling, the following anchor boxes for our multi-tasks are predicted:

$$
\left(p_{o d}^{w}, p_{o d}^{h}\right) \in\{(180,180),(120,120),(60,60)\} .
$$

OD with reasoning are performed across scales of $\times 1, \times 2$ and $\times 4$ and GD are performed across scales of $\times 1$ and $\times 2$. Therefore, 9 anchor boxes are predicted with 4 bounding box offsets, object probability, object class (class number) and (class number +1$) \times 2$ reasoning classes $(\mathrm{FC}, \mathrm{CC})$ for $\mathrm{OD}$ with reasoning. In addition, 8 anchor boxes are predicted with 4 bounding box offsets, orientation, grasp probability and object class (class number) for GD.

\section{Reasoning post-processing: from class to index}

Fig. 3 illustrates the differences between the works of Zhang [1], [2] and our proposed methods. Previous works generated necessary information for OD with reasoning and GD. Deep neural networks (N) generated local features or OD or GD or relationship among objects (FC, CC), respectively and sequentially. However, as shown in the work of Redmon [5], dealing with GD and classification often improves the overall performance of GD. We propose a novel single network $(\mathrm{N})$ to yield most information on $\mathrm{OD}$ and GD with simple reasoning post-processing $(\mathrm{P})$ for building hierarchy among objects. 


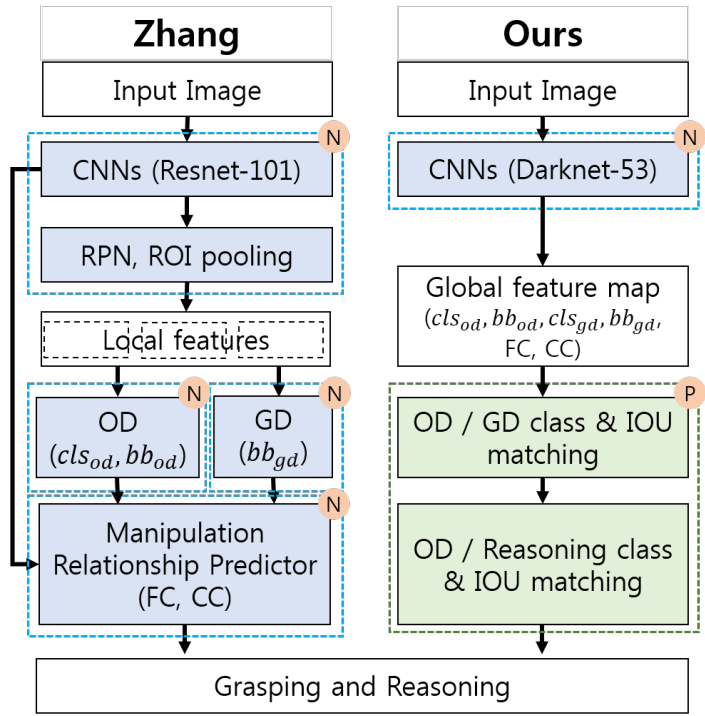

Fig. 3: Schematic pipelines of Zhang [1], [2] vs ours.

For a generated global feature map including class information, bounding box information and FC / CC, reasoning post-processing can build index relationships using class information. Firstly, non-maximum suppression is applied to GD and OD to eliminate unnecessary detection results. Secondly, grouping of bounding boxes in OD and GD $\left(b b_{o d}\right.$, $\left.b b_{g d}\right)$ is performed based on their class information $\left(\mathrm{cls}_{\text {od }}\right.$, $\left.c l s_{g d}\right)$. Then, the spatial information of bounding boxes are used for further grouping bounding box pairs for OD and GD based on the IOU (Intersection Over Union) as follows:

$$
I O U=\frac{b b_{o d} \cap b b_{g d}}{b b_{o d} \cup b b_{g d}} .
$$

Lastly, among GD candidates whose IOU exceeds a certain threshold, the best probability for GD is selected to obtain the final OD / GD bounding box pair.

Similarly, we compare object classes with child classes already obtained in the model to get the relationships between them by matching boxes with IOU threshold. With this, we can make a object relation graph for robot grasping.

\section{E. Loss function for multi-task $G D, O D$, reasoning}

For the output vectors OD, GD and R of DNN and the ground truth (GT) $O D_{g t}, G D_{g t}$ and $R_{g t}$, we propose the follow loss function to train our single multi-task DNN:

$$
\begin{aligned}
& \sum_{i \in \Omega} \sum_{j \in\{o d, g d\}}\left\{\sum_{k \in\{x, y, w, h\}} \operatorname{MSE}\left(k_{j}^{i}, k_{j, g t}^{i}\right)+\right. \\
& \left.\sum_{k \in p r}\left(-\log k_{j}^{i}\right)+\sum_{k \in c l s_{o b}} \operatorname{FocLoss}\left(k_{j}^{i}, k_{j, g t}^{i}\right)\right\}+ \\
& \lambda_{\mathrm{n}} \sum_{i \in \Omega^{c}} \sum_{j \in\{o d, g d\}} \sum_{k \in p r}\left(-\log \left(1-k_{j}^{i}\right)\right)+ \\
& \sum_{i \in \Omega} \sum_{j \in R} \sum_{k \in c l s_{f c}, c l s_{c c}} \operatorname{BiFocLoss}\left(k_{j}^{i}, k_{j, g t}^{i}\right)+ \\
& \sum_{i \in \Omega} \sum_{j \in g d} \sum_{k \in \theta} \operatorname{MSE}\left(k_{j}^{i}, k_{j, g t}^{i}\right)
\end{aligned}
$$

where $x, y, w, h, z$ are functions of $t^{x}, t^{y}, t^{w}, t^{h}, t^{z}$ respectively, $\Omega$ is the grid cells where the object or grasping object are located. Since $c l s_{f c}$ and $c l s_{c c}$ are multi-classes, we used binary focal loss (BiFocLoss) [30]. Focal loss gamma is set to 2 and we set $\lambda_{\mathrm{n}}=100$.

\section{EXPERIMENTAL EVALUATIONS}

We evaluated our proposed methods on the VMRD dataset [1], the Cornell dataset [3], real robot grasping of single novel objects with a 4-axis robot arm and real multitask robot grasping of multiple novel objects in various scenes with a 7-axis Baxter robot. A RGB-D camera (Intel RealSense D435) was installed and used to have the fieldof-view including robot and workspace from the top.

\section{A. Implementation details}

Darknet-53 was also implemented used for the evaluations on the VMRD and for real multi-task robot grasping of multi objects. Darknet-19 was implemented and used for other evaluations. Either stochastic gradient descent (SGD) with momentum of 0.9 or Adam optimizer was used for training. Learning rate was 0.001 and mini batch size was set to 2 . For self-evaluation to optimize the model, total epoch was 50. Once the model is optimized, total epoch was set to 100 with reducing learning rate by half every 30 epochs. Patch based training was performed with the sizes of $608 \times 608$ using data augmentation [1]. All algorithms were tested on the platform with a single GPU (NVIDIA GTX1080Ti),a single CPU (Intel i7-7700K 4.20GHz) and 32GB memory.

\section{B. Evaluations on VMRD and Cornell datasets}

We performed benchmarks using the Cornell dataset [3], [4] as illustrated in Fig. 4b. This dataset consists of 855 images (RGB-D) of 240 different objects with GT labels of a few graspable / non-graspable rectangles. We cropped images with $360 \times 360$, but did not resize it to $224 \times 224$. Five-fold cross validation $(\mathrm{CV})$ was performed and average prediction accuracy was reported for image-wise and object-wise splits. When the difference between the output orientation $\theta$ and the GT orientation $\theta_{g t}$ is less than a certain threshold (e.g., $30^{\circ}$ ), then IOU that is larger than a certain threshold (e.g., $0.25,0.3$ ) will be considered as a successful grasp detection. The same metric for accuracy has been used in previous works [4], [5], [31], [32], [6], [7], [8], [2].

VMRD dataset was used to train our single multi-task network. VMRD consists of 4233 train data and 450 test data (RGB images) as illustrated in Fig. 4a In this dataset, there are 2-5 objects stacked in each image and GT for OD with with class label \& relationship index, GD with class label and FC / CC labels. There are 31 object classes. If the IOU for predicted OD and GT OD is larger than 0.5 and the best grasping point for that object meets the above Cornell evaluation metric, it is considered as success (mAP with grasp or $\mathrm{mAPg}$ ) [1]. 

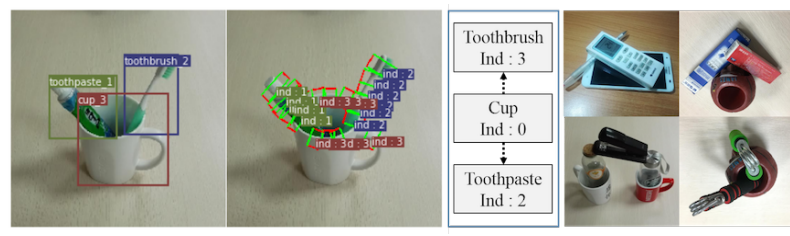

(a)

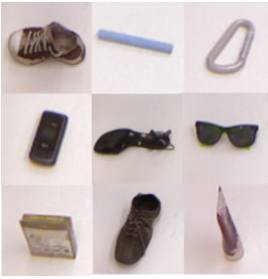

(b)

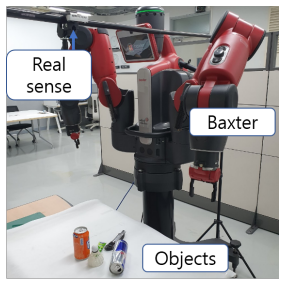

(c)

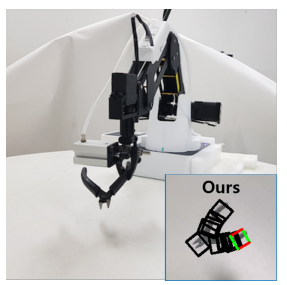

(d)
Fig. 4: (a) VMRD dataset. (b) images from Cornell dataset. (c) our real multi-task evaluation environment (Baxter). (d) our robot grasping experiment with 4-axis robot.

\section{Evaluation of multi-tasks $O D, G D$, reasoning with Baxter}

We evaluated our proposed methods using a Baxter with 7-axis arms (Rethink Robotics, Germany, see Fig. 4c for three different scenarios of cluttered scene, stacking scene and complex invisible stacking scene. In cluttered scene, it was recorded as success if the robot grasped the target in a single try. In stacking and invisible scenes, it was recorded as success if the robot removed objects over the target and then grasp the target. For the invisible scene with no target detected, the robot put away overlapping objects one by one until the target is found. Prediction was performed separately between all robot movements. All combinations of items, the target object and stacking orders are chosen randomly.

\section{Evaluation of GD with 4-axis robot arm}

We evaluated our proposed methods with a small 4axis robot arm (Dobot Magician, Shenzhen YueJiang Tech, China) for novel object grasping. The following 8 novel objects (toothbrush, candy, earphone cap, cable, styrofoam bowl, L-wrench, nipper, pencil) were used for grasping tasks. If the robot gripper grasps an object and moves the object to another place, it is counted as success.

\section{RESULTS}

\section{A. Simulation results on VMRD dataset}

Table II summarizes our ablation study results on the VMRD multi-task robot grasp dataset. The method on the first row of Table I] is an initial extension of YOLOv3 to multi-task robot grasping. Then, by changing activation function, scale, loss function and optimization algorithm, we

TABLE I: Self-evaluation summary on VMRD.

\begin{tabular}{|c|c|c|c|c|}
\hline Across scales & Activation & Loss & Opt. & mAPg (\%) \\
\hline $1,2,3$ & Sigmoid & Cross Entropy & Adam & 56.5 \\
$1,2,3$ & Softmax & Cross Entropy & Adam & 63.1 \\
1,2 & Softmax & Cross Entropy & Adam & 64.9 \\
1,2 & Softmax & Focal Loss & Adam & 67.1 \\
1,2 & Softmax & Focal Loss & SGD & 69.2 \\
\hline
\end{tabular}

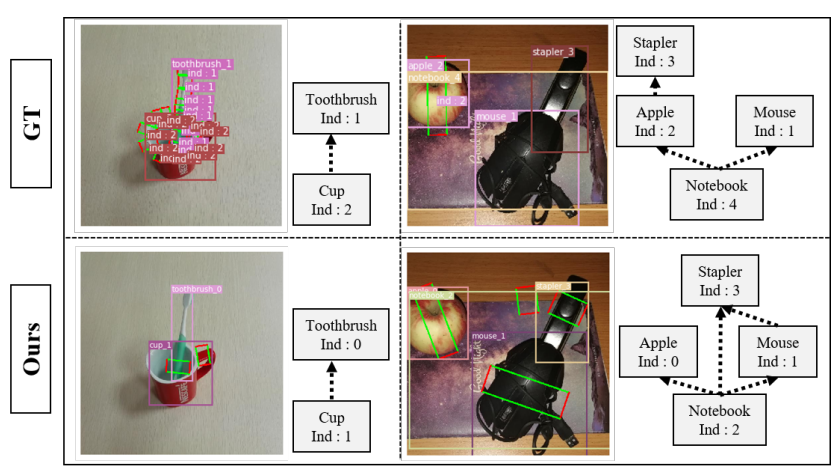

(a)

(b)

Fig. 5: Multi-task detection results for VMRD. The $1^{\text {st }}$ row is GT and the $2^{\text {nd }}$ row is the results of our proposed methods. Note that our method yielded correct reasoning result for "Stapler" while GT incorrectly describes it.

were able to optimize our single DNN for multi-task OD, GD and reasoning from $56.5 \% \mathrm{mAPg}$ (mAP with grasp) to $69.2 \% \mathrm{mAPg}$. Note that focal loss is often used for predicting unbalanced object classes [30]. The VMRD dataset seems unbalanced since there are 2061 notebooks and 93 chargers. Focal Loss gives small weights to well-classified examples while gives large weights to some examples that are difficult to classify to focus on learning difficult examples.

Fig. 5 illustrates qualitative results for generating multitask robotic grasps. Fig. 5(a) shows a two-level stacking case and its OD, GD and reasoning results of our proposed method (bottom row) and GT (top row). Fig. 5(b) shows another multi-stacking case of GT (top) and the output of our proposed method (bottom). Note that GT contains an error in reasoning (Stapler is not on the Apple) while our method corrected for it through training on many examples.

Table $\Pi$ summarizes the results of the results of previous methods [1], [2] and our proposed method. Our proposed method yielded state-of-the-art performance of $74.3 \% \mathrm{mAP}$ with grasp $(\mathrm{mAPg})$ at the fastest computation speed of 33.3 FPS for a high resolution input image $(608 \times 608)$.

TABLE II: Performance summary on VMRD dataset.

\begin{tabular}{|l|c|c|}
\hline Method & mAPg (\%) & Speed (FPS) \\
\hline Zhang [2] baseline, OD, GD & 54.5 & 10.3 \\
Zhang [2], OD, GD & 68.2 & 9.1 \\
Zhang [1], OD, GD, reasoning & 70.5 & 6.5 \\
\hline Ours, OD, GD, reasoning & 74.6 & 33.3 \\
\hline
\end{tabular}

\section{B. Simulation results on Cornell dataset}

Fig. 6 illustrates qualitative results for generating robotic grasps using our methods without and with predictions across scales. Both yielded fairly good grasp detection results, but there were often cases with fine details where predictions across scales improved the results such as the case with scissors as shown in Fig 6

Table III summarizes the results of previous methods and our methods. Our proposed method with RGB-D yielded state-of-the-art performance of up to $98.6 \%$ prediction accuracy for image-wise split and up to $97.2 \%$ for object- 


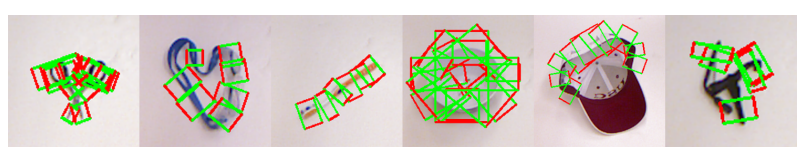

(a) Ground truth

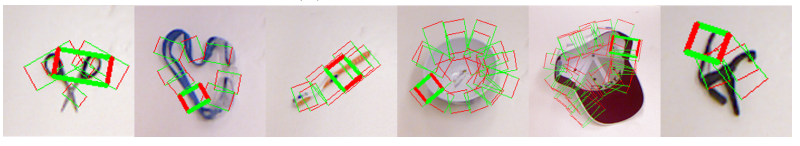

(b) Ours without predictions across scales

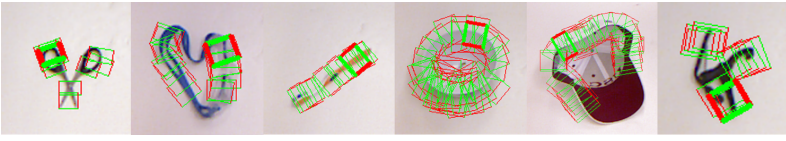

(c) Ours with predictions across scales (proposed)

Fig. 6: GD results on Cornell dataset using our methods without and with predictions across scales.

wise split, respectively. Our proposed method with RGB also yielded comparable results to state-of-the-art methods. Note that our proposed method yielded these results with the smallest DNN and the fastest computation time of $16 \mathrm{~ms}$ per high resolution image $(360 \times 360)$ that can be potentially useful for real-time applications or stand-alone applications with limited memory and energy. Using depth and predictions across scales improved performance.

TABLE III: Summary on Cornell data (25\% IOU).

\begin{tabular}{|l|c|c|c|c|}
\hline Method & Input & $\begin{array}{c}\text { Image } \\
(\%)\end{array}$ & $\begin{array}{c}\text { Object } \\
(\%)\end{array}$ & $\begin{array}{c}\text { Speed } \\
\text { (FPS) }\end{array}$ \\
\hline Lenz [4], SAE & RGB-D & 73.9 & 75.6 & 0.08 \\
Redmon [5], Alexnet & RG-D & 88.0 & 87.1 & 13.2 \\
Kumra [31], Resnet-50 & RGB-D & 89.2 & 88.9 & 16 \\
Asif [32] & RGB-D & 90.2 & 90.6 & 41 \\
Guo [6] \#a, ZFnet & RGB-D & 93.2 & 82.8 & - \\
Guo [6] \#c, ZFnet & RGB-D & 86.4 & 89.1 & - \\
Chu [7], Resnet-50 & RG-D & 96.0 & 96.1 & 8.3 \\
Zhou [8], Resnet-50 & RGB & 97.7 & 94.9 & 9.9 \\
Zhou [8], Resnet-101 & RGB & 97.7 & 96.6 & 8.5 \\
Zhang [2], Resnet-101 & RGB & 93.6 & 93.5 & 25.2 \\
\hline Ours, Darknet-19 & RGB & 97.7 & 96.1 & 140 \\
Ours, Darknet-19 & RG-D & 98.6 & 97.2 & 62.5 \\
\hline
\end{tabular}

C. Results of multi-task $O D, G D$, reasoning with Baxter

Fig. 7a shows the OD, GD and reasoning results of our proposed methods for different scenarios such as (a) cluttered scene (CS), (b) stacking scene (SS) and (c) invisible scene (IS). For CS, the target "Stapler" was successfully located with proper grasp. For SS, the target "Knife" and its related object "Toothpaste" were well located with correct relationship reasoning. For IS, the target was not detected due to occlusion, but as overlapped objects are removed based on the reasoning results (green arrow), the target was finally detected at the step 3 . Table $[\mathrm{IV}$ shows the performance

TABLE IV: Performance summary of grasping tasks for cluttered (CS), stacking (SS) and invisible (IS) scenes.

\begin{tabular}{|c|c|c|c|c|}
\hline \#objects & 2 & 3 & 4 & 5 \\
\hline CS & - & $86.7(13 / 15)$ & $85.0 \%(17 / 20)$ & $86.7 \%(26 / 30)$ \\
\hline SS & $80.0 \%(8 / 10)$ & $60.0 \%(9 / 15)$ & $55.0 \%(11 / 20)$ & - \\
\hline IS & - & $60.0 \%(9 / 15)$ & $40.0 \%(8 / 20)$ & $28.0 \%(7 / 25)$ \\
\hline
\end{tabular}

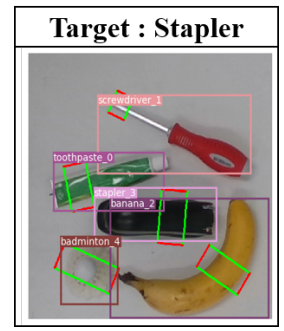

(a) Cluttered scene

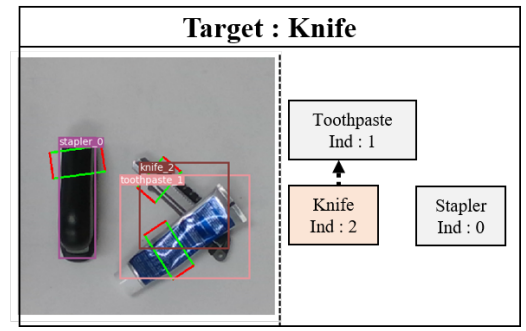

(b) Stacking scene

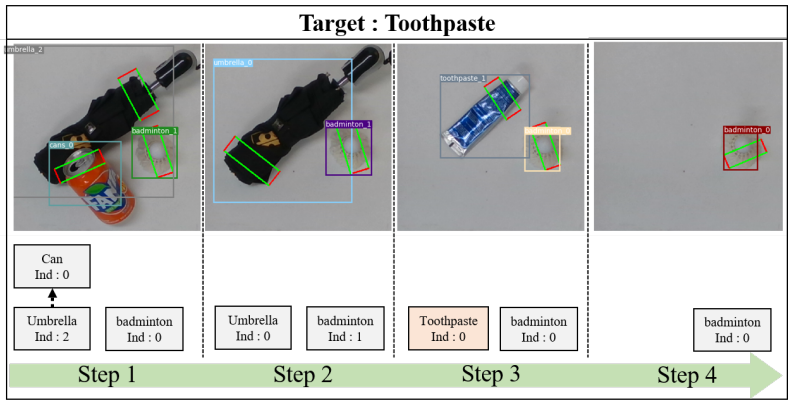

(c) Invisible scene

Fig. 7: Target grasp detection results in (a) cluttered scene, (b) stacking scene and (c) challenging invisible scene.

summary of the results of our proposed method with a Baxter robot. In CS, the accuracy was high, up to $86.7 \%$ regardless of the increase in the number of objects. However, in SS, we observed that increasing the number of objects decreases grasp success rate possibly due to the difficulties of FC, $\mathrm{CC}$ predictions among them with severe occlusions. This phenomenon was also observed in challenging IS case.

\section{Results of GD with 4-axis robot arm}

Fig. 4d illustrates our robot grasp experiment with "nipper". Note that due to small gripper and small objects, grasp detection accuracy was important for successful robot grasping. Our proposed method yielded $95.3 \%$ mean grasp success rate with $6.5 \%$ standard deviation for 8 novel, small objects with 8 repetitions per each object.

\section{CONCLUSIONS}

We propose a single multi-task DNN that yields the information on GD, OD and reasoning among objects with a simple post-processing. Our proposed methods yielded state-of-the-art performance with the accuracy of $98.6 \%$ and $74.2 \%$ and the computation speed of 33 and 62 FPS on VMRD and Cornell datasets, respectively. Our methods also yielded $95.3 \%$ grasp success rate for single novel object grasping with a 4 -axis robot arm and $86.7 \%$ grasp success rate in cluttered novel objects with a Baxter robot.

\section{ACKNOWLEDGMENT}

This work was supported by the Technology Innovation Program or Industrial Strategic Technology Development Program (10077533, Development of robotic manipulation algorithm for grasping/assembling with the machine learning using visual and tactile sensing information) funded by the Ministry of Trade, Industry \& Energy (MOTIE, Korea). 


\section{REFERENCES}

[1] Hanbo Zhang, Xuguang Lan, Lipeng Wan, Chenjie Yang, Xinwen Zhou, and Nanning Zheng. Rprg: Toward real-time robotic perception, reasoning and grasping with one multi-task convolutional neural network. arXiv preprint arXiv:1809.07081, 2018.

[2] Hanbo Zhang, Xuguang Lan, Site Bai, Xinwen Zhou, Zhiqiang Tian, and Nanning Zheng. Roi-based robotic grasp detection for object overlapping scenes. arXiv preprint arXiv:1808.10313, 2018.

[3] Ian Lenz, Honglak Lee, and Ashutosh Saxena. Deep Learning for Detecting Robotic Grasps. In Robotics: Science and Systems, page P12, June 2013.

[4] Ian Lenz, Honglak Lee, and Ashutosh Saxena. Deep learning for detecting robotic grasps. The International Journal of Robotics Research, 34(4-5):705-724, April 2015.

[5] J Redmon and A Angelova. Real-time grasp detection using convolutional neural networks. In IEEE International Conference on Robotics and Automation (ICRA), pages 1316-1322, 2015.

[6] Di Guo, Fuchun Sun, Huaping Liu, Tao Kong, Bin Fang, and Ning $\mathrm{Xi}$. A hybrid deep architecture for robotic grasp detection. In IEEE International Conference on Robotics and Automation (ICRA), pages 1609-1614, 2017.

[7] Fu-Jen Chu, Ruinian Xu, and Patricio A Vela. Real-World Multiobject, Multigrasp Detection. IEEE Robotics and Automation Letters, 3(4):3355-3362, October 2018.

[8] Xinwen Zhou, Xuguang Lan, Hanbo Zhang, Zhiqiang Tian, Yang Zhang, and Narming Zheng. Fully convolutional grasp detection network with oriented anchor box. In 2018 IEEE/RSJ International Conference on Intelligent Robots and Systems (IROS), pages 72237230. IEEE, 2018.

[9] Hanbo Zhang, Xuguang Lan, Xinwen Zhou, Zhiqiang Tian, Yang Zhang, and Nanning Zheng. Visual manipulation relationship network for autonomous robotics. In 2018 IEEE-RAS 18th International Conference on Humanoid Robots (Humanoids), pages 118-125. IEEE, 2018.

[10] Joseph Redmon and Ali Farhadi. Yolov3: An incremental improvement. arXiv preprint arXiv:1804.02767, 2018.

[11] Jeannette Bohg, Antonio Morales, Tamim Asfour, and Danica Kragic. Data-Driven Grasp Synthesis-A Survey. IEEE Transactions on Robotics, 30(2):289-309, March 2014.

[12] Ashutosh Saxena, Justin Driemeyer, and Andrew Y Ng. Robotic grasping of novel objects using vision. The International Journal of Robotics Research, 27(2):157-173, February 2008.

[13] Yun Jiang, Stephen Moseson, and Ashutosh Saxena. Efficient grasping from RGBD images: Learning using a new rectangle representation. In IEEE International Conference on Robotics and Automation (ICRA), pages 3304-3311, 2011.

[14] Zhichao Wang, Zhiqi Li, Bin Wang, and Hong Liu. Robot grasp detection using multimodal deep convolutional neural networks. Advances in Mechanical Engineering, 8(9):1-12, September 2016.

[15] Jeffrey Mahler, Jacky Liang, Sherdil Niyaz, Michael Laskey, Richard Doan, Xinyu Liu, Juan Aparicio Ojea, and Ken Goldberg. Dex-Net 2.0: Deep Learning to Plan Robust Grasps with Synthetic Point Clouds and Analytic Grasp Metrics. In Robotics: Science and Systems (RSS), 2017.

[16] A Krizhevsky, I Sutskever, and G E Hinton. Imagenet classification with deep convolutional neural networks. In Advances in Neural Information Processing Systems 25, pages 1097-1105, 2012.

[17] Kaiming He, Xiangyu Zhang, Shaoqing Ren, and Jian Sun. Deep Residual Learning for Image Recognition. In IEEE Conference on Computer Vision and Pattern Recognition (CVPR), pages 770-778, 2016.

[18] Shaoqing Ren, Kaiming He, Ross Girshick, and Jian Sun. Faster R-CNN: Towards real-time object detection with region proposal networks. In Advances in Neural Information Processing Systems 28, pages 91-99, 2015.

[19] Umar Asif, Jianbin Tang, and Stefan Harrer. GraspNet: An Efficient Convolutional Neural Network for Real-time Grasp Detection for Lowpowered Devices. In International Joint Conference on Artificial Intelligence (IJCAI), pages 4875-4882, 2018.

[20] Edward Johns, Stefan Leutenegger, and Andrew J Davison. Deep learning a grasp function for grasping under gripper pose uncertainty. In IEEE International Conference on Intelligent Robots and Systems (IROS), pages 4461-4468. IEEE, 2016.
[21] Jeffrey Mahler, Matthew Matl, Xinyu Liu, Albert Li, David Gealy, and Ken Goldberg. Dex-Net 3.0: Computing Robust Vacuum Suction Grasp Targets in Point Clouds Using a New Analytic Model and Deep Learning. In IEEE International Conference on Robotics and Automation (ICRA), pages 5620-5627. IEEE, May 2018.

[22] Ulrich Viereck, Andreas ten Pas, Kate Saenko, and Robert Platt. Learning a visuomotor controller for real world robotic grasping using simulated depth images. In Conference on Robot Learning (CoRL), pages 291-300, 2017.

[23] Douglas Morrison, Peter Corke, and Jürgen Leitner. Closing the loop for robotic grasping: A real-time, generative grasp synthesis approach. arXiv preprint arXiv:1804.05172, 2018.

[24] Kourosh Khoshelham and Sander Oude Elberink. Accuracy and Resolution of Kinect Depth Data for Indoor Mapping Applications. Sensors, 12(2):1437-1454, February 2012.

[25] Lerrel Pinto and Abhinav Gupta. Supersizing self-supervision: Learning to grasp from $50 \mathrm{~K}$ tries and 700 robot hours. In IEEE International Conference on Robotics and Automation (ICRA), pages 3406-3413. IEEE, May 2016.

[26] Wei Liu, Dragomir Anguelov, Dumitru Erhan, Christian Szegedy, Scott Reed, Cheng-Yang Fu, and Alexander C Berg. Ssd: Single shot multibox detector. In European conference on computer vision, pages 21-37. Springer, 2016.

[27] Joseph Redmon and Ali Farhadi. YOLO9000: Better, Faster, Stronger. In IEEE Conference on Computer Vision and Pattern Recognition (CVPR), pages 6517-6525, 2017.

[28] Xuanchen Zhang, Yuntao Song, Yang Yang, and Hongtao Pan. Stereo vision based autonomous robot calibration. Robotics and Autonomous Systems, 93:43-51, 2017.

[29] Tsung-Yi Lin, Piotr Dollár, Ross Girshick, Kaiming He, Bharath Hariharan, and Serge Belongie. Feature pyramid networks for object detection. In Proceedings of the IEEE Conference on Computer Vision and Pattern Recognition, pages 2117-2125, 2017.

[30] Tsung-Yi Lin, Priya Goyal, Ross Girshick, Kaiming He, and Piotr Dollár. Focal loss for dense object detection. In Proceedings of the IEEE international conference on computer vision, pages 2980-2988, 2017.

[31] Sulabh Kumra and Christopher Kanan. Robotic grasp detection using deep convolutional neural networks. In IEEE International Conference on Intelligent Robots and Systems (IROS), pages 769-776, 2017.

[32] Umar Asif, Mohammed Bennamoun, and Ferdous A Sohel. RGB-D Object Recognition and Grasp Detection Using Hierarchical Cascaded Forests. IEEE Transactions on Robotics, 33(3):547-564, May 2017. 